\title{
Los pasos de La Llorona sobre Ciudad Juárez: voces de resistencia en la literatura de mujeres de la frontera México-Estados Unidos*
}

Este estudio analiza un corpus de literatura de la frontera norte de México escrita por mujeres en relación a la violencia de Ciudad Juárez, con el objetivo de identificar representaciones de la figura histórica y mítica de La Llorona desde la interpretación cultural chicana. La propuesta crítica de autoras como Gloria Anzaldúa acerca de una identidad transgresora, conformada a partir del arquetipo de la Madre terrible, permite reconocer significaciones positivas de las voces mexicanas femeninas vinculadas al dolor, el silencio, el llanto y el grito, para establecer un discurso colectivo de resistencia y empoderamiento contra la violencia y el olvido.

Palabras clave: literatura, La Llorona, mujeres, violencia, resistencia, Ciudad Juárez, chicana.

This study analyzes a literary corpus from the northern border of Mexico written by women in relation to violence in Ciudad Juárez, with the purpose of identifying representations of the historical and mythical figure of La Llorona from the point of view of Chicana cultural interpretation. Critical proposals by authors such as Gloria Anzaldúa, about a transgressing identity build upon the archetype of "the terrible mother" allows to recognize positive meanings in female Mexican voices associated to pain, silence, crying and shouting, in order to establish a collective discourse of resistance and empowerment against violence and forgetfulness.

Keywords: literature, La Llorona, women, violence, resistance, Ciudad Juárez, chicana.

Una parte significativa de la literatura reciente escrita por mujeres en torno a la violencia de Ciudad Juárez inscribe un acto de ruptura de las víctimas contra el silencio impuesto por la agresión del sistema capitalista, el dominio patriarcal y la impunidad del Estado mexicano. En ambos lados de la frontera mexicana-estadounidense la poesía y la narrativa interpretan experiencias de subordinación y casos de violencia que afectan 
principalmente a las mujeres. Las escritoras de dichos espacios recurren a un discurso reivindicativo que busca intervenir en las plataformas sociales y políticas de México y de Estados Unidos, desde las cuales puedan ejercer poder sobre sus cuerpos.

Desde 1993, Ciudad Juárez ha constituido un foco de atención internacional debido al número de feminicidios que ocurren entre esta ciudad y El Paso, Texas. Hasta el año 2003, Amnistía Internacional concluyó que habían sido asesinadas 370 mujeres jóvenes de origen mexicano en Ciudad Juárez y Chihuahua (Gaspar de Alba y Guzmán 65). Actualmente siguen desapareciendo mujeres, algunas de ellas son encontradas muertas en la periferia y en el desierto. Madres y familias, junto con organizaciones civiles y activistas, encabezan una serie de investigaciones para hallar respuestas y hacer justicia. En el libro Making a Killing. Femicide, Free Trade and La Frontera, editado y publicado en 2010 por Alicia Gaspar de Alba y Georgina Guzmán, diferentes autoras exponen sus opiniones acerca de las posibles razones de dichos crímenes. En la mayoría de los ensayos se denuncia la impunidad de las autoridades de México y de Estados Unidos. En resumen, Elvia Arriola argumenta que tanto las maquiladoras como el gobierno mexicano son responsables; éste último desde el momento en que favorece a los inversionistas de las fábricas, antes que a las obreras (25-6I). Alicia Gaspar de Alba menciona que las maquiladoras ponen a las mujeres en una situación de trabajo deshumanizante, al priorizar la eficiencia de producción, fomentan las condiciones precarias en su traslado a la fábrica y en la infraestructura de sus lugares de vivienda, exponiéndolas al peligro y a su muerte (63-93). Además, las autoras señalan el machismo y la misoginia como una de las principales causas de los crímenes. María Socorro Tabuenca examina la visión de Juárez como una ciudad perversa donde se difunde la imagen negativa de las mujeres, en su posición de trabajadoras y transgresoras (95-II9). Junto con Arriola se refiere al desafortunado juicio que las culpabiliza, pues son consideradas prostitutas o acusadas de vestir en forma provocativa y, en ese sentido, señaladas como responsables de su muerte. Por último, en el mismo volumen, Jane Caputi propone que una posible razón de los crímenes es la enseñanza misógina de antiguos mitos aztecas, que sobrevive en valores y creencias mexicanas actuales. Específicamente, el mito de Coyolxauhqui, diosa desmembrada por su hermano Huitzilopochtli - como se explica más adelante -, se actualiza en los asesinatos de mujeres en Ciudad Juárez para reafirmar la supremacía del poder masculino (279294). De tales hechos e hipótesis se ocupan los textos literarios de mujeres del norte de México y chicanas que motivan el presente análisis. 
En Estados Unidos, la literatura chicana ha desarrollado un sólido corpus literario y teórico-crítico que problematiza la presencia de elementos claves tanto de la historia como del imaginario mexicano en su cultura, y que a la vez hacen parte de un ejercicio activo de afirmación de su mestizaje y subjetividad. Entre ellos, las figuras arquetípicas de la Madre de los relatos precolombinos y del periodo de colonización española funcionan como referentes para comprender las configuraciones identitarias de los sujetos femeninos. En el caso de la literatura de la frontera norte de México escrita por mujeres, concentrada mayoritariamente en Tijuana y Ciudad Juárez, es menos prolífica la producción en este sentido. Si bien se está conformando un importante corpus literario, es más limitado el desarrollo teórico y crítico que lo aborda.

El propósito es revisar una selección de textos poéticos y narrativos de autoras de la frontera norte de México y chicanas con la finalidad de identificar aspectos simbólicos asociados a La Llorona, figura vinculada a la diosa Coatlicue y a la Malinche en los relatos históricos y míticos del México precolombino. De manera específica, el objetivo es realizar un análisis enfocado en las experiencias de llanto asociado a las prácticas reales y simbólicas de errancia, búsqueda, silenciamiento y resistencia, mediante las cuales se representan voces de las mujeres que reclaman la pérdida o muerte de sus hijas u otras mujeres en medio de los escenarios de violencia registrados en Ciudad Juárez durante las últimas décadas. Estas claves se recuperan en el análisis de relatos y poemas de las escritoras Arminé Arjona, Araceli Bobadilla, Rosina Conde, Dolores Dorantes, Elia Hatfield y Micaela Solís. Se incluyen en este estudio porque son originarias de la frontera norte de México, radican en Ciudad Juárez, o bien, han escrito sobre esta última ciudad, como la tijuanense Rosina Conde. Se pretende recuperar en sus obras elementos distintivos de la imagen simbólica de La Llorona, en diálogo con su representación dentro de la literatura chicana. Principalmente se realiza una lectura de la obra de Gloria Anzaldúa, escasamente difundida en México. El propósito es alumbrar e interpretar posibles identidades desde las cuales se configuran y afirman sujetos femeninos de resistencia, dentro del contexto de violencia en la zona de cruce fronteriza o borderland mexicanaestadounidense. ${ }^{\mathrm{I}}$

Si bien la referencia a La Llorona no aparece de manera explícita en los textos de las autoras sobre Ciudad Juárez, subyacen rasgos de ésta en la enunciación de las voces femeninas. Se advierten a partir de dos condiciones: primero, a través de las expresiones de llanto de las mujeres en su manifestación de dolor, en la descripción de una situación de 
errancia, sobre un espacio de represión y silenciamiento y, por otra parte, mediante un llanto de resistencia que actúa contra el silencio, generando una búsqueda colectiva, transformado en grito y fuerza creativa. El abordaje de estas perspectivas en detalle permite reconocer diversas significaciones de la figura de La Llorona retomadas por las autoras chicanas, especialmente en relación a la imagen de la mujer/madre que traiciona a su cultura, es víctima de una muerte violenta, se encuentra errante buscando a sus hijos muertos, vuelve como un espíritu angustiado o habla en memoria de las muertas o desaparecidas.

De acuerdo con la doble perspectiva de las chicanas, La Llorona aparece en parte como una figura simbólica de culpa y opresión, mujer y madre castigada, pero, principalmente, como modelo femenino que lucha contra la cultura dominante, se apropia de su cuerpo, de su voz, y se libera. Anzaldúa desarrolla inicialmente dichas pautas en su libro Borderlands/La Frontera: The New Mestiza (1987). Bajo su lectura, en la literatura de las mencionadas autoras de la frontera del norte de México, estas claves sustentan el discurso de empoderamiento de las mujeres contra la violencia propiciada por efectos del machismo, el sistema patriarcal y la impunidad del Estado, que se deslinda de su responsabilidad de hacer justicia a las muertas o desaparecidas.

Con la voluntad de desarrollar un estudio literario metodológicamente "bifronterizo", este análisis busca incorporarse a los debates que establecen diversas escritoras y críticas literarias chicanas y mexicanas del norte, centro y sur del país, en torno a la incomunicación, diálogos posibles o comparaciones entre las literaturas de ambos espacios fronterizos. ${ }^{2}$ En el coloquio del cual surgió el texto Las formas de nuestras voces: Chicana and Mexicana Writers in Mexico, escritoras enfocadas en el estudio de ambas fronteras se ocuparon de discutir acerca de la necesidad de crear un "terreno discursivo" (Joysmith 38) donde confluyan y dialoguen las dos literaturas. Aralia López manifiesta la pertinencia de estudiar la escritura de mujeres chicana y mexicana desde una perspectiva crítica feminista para "observar cómo estas abordan, en cuanto mujeres, las tensiones históricas provenientes de sus contextos nacionales; $\mathrm{y}$, en cuanto escritoras propiamente, cómo se articulan a la tradición literaria que las precede" (53).

Así, tras identificar conexiones entre ambas literaturas en relación al imaginario sobre La Llorona, se recurre a antecedentes literarios de las escritoras Chicanas Gloria Anzaldúa, Cherríe Moraga, Sandra Cisneros, Ana Castillo, y Alicia Gaspar de Alba. 3 La Llorona constituye para ellas un símbolo de tradición y sustento cultural mediante el cual refuerzan los vínculos con su origen mexicano. Su reescritura forma parte de una 
tradición compartida por la comunidad chicana. Las mujeres, y también los hombres que participan dentro de lo que conforma el movimiento cultural chicano, recuperan este relato para entender su mestizaje, así como las secuelas de la ruptura con sus otros mexicanos y estadounidenses. Los latinos, señala Antonia Castañeda, escuchan todavía su voz: "Like a blurred, cracked photograph her image is retained. She [La Llorona] is etched in the very center of our consciousness" (97-98). Según Pilar Melero, las mexicanas, refiriéndose también a las chicanas, elaboran su discurso desde las faldas de la madre. Ellas "have sought refuge in the image of the mother to construct themselves as political, historic and literary subjects" (6).

Junto con Coatlicue, madre de los dioses aztecas, Coyolxauhqui, su hija rebelde, y Malinalli o la Malinche, indígena, intérprete y amante de Hernán Cortés durante la colonización española, La Llorona es arquetipo de la Madre terrible. La caracterización de La Llorona, como señala Cristina Santos, forma parte del arquetipo de la Madre terrible o maligna Maligned Mother(hood) - presente en diversas culturas. La primera referencia en México surge en la mitología indígena anterior a la colonización española, a través de la diosa azteca Coatlicue, asesinada por su hijo Huitzilopochtli. La diosa de falda de serpientes, protectora de la fetilidad y del legado de su pueblo, fue considerada monstruo desde la perspectiva de los colonizadores porque no reflejaba la figura de madre ejemplar, de acuerdo con el orden patriarcal y cristiano (64-65). Para la cultura dominante el desmembramiento termina con el poder maléfico de la diosa manifestado en la apariencia horrorosa de su cuerpo. Sin embargo, la visión indígena, misma que se expone en el Museo Nacional de Antropología e Historia de México, resalta su fuerza y afirma la expresión de su sexualidad (66).4 Así mismo, la Llorona tiene su origen en la figura de Cihuacoatl, diosa mexica de la fertilidad, también demonizada por el discurso colonial, pues contradice los ideales de sexualidad asociados al cristianismo. Cihuacoatl es una versión adecuada para este análisis, pues no asesina a sus hijos, sino que predice la caída de los indígenas frente a los españoles y se lamenta. El relato la describe apareciéndose a Moctezuma I en Tenochtitlan, vestida de blanco y llorando el abandono que les espera a sus hijos (68).

La representación de la Llorona en la literatura chicana se introduce clara e intencionada como forma de resistencia frente a la cultura hegemónica, legitima o afirma las subjetividades, la mezcla de rasgos culturales que las constituyen, y es fundamento de lo que Gloria Anzaldúa reconoce como la "conciencia mestiza": 
Mi identidad chicana está forjada en la historia de la resistencia de la mujer india. Los rituales de luto de la mujer azteca eran ritos de desafío para protestar contra los cambios culturales que rompieron la igualdad y el equilibrio entre mujeres y varones, y protestar contra su desplazamiento a un estatus inferior, su denigración. Como la Llorona el único medio de protesta de la mujer india era el lamento. (Los Movimientos 78)

Las escritoras de la frontera norte de México y las chicanas recuperan de manera distinta el imaginario de La Llorona. Está ausente en la configuración de las identidades de las primeras, según las lecturas realizadas, pues no ha dado pie a un discurso sobre la conciencia mestiza y a la definición de figuras contestatarias. Del lado de la crítica mexicana, Marisa Belausteguigoitia advierte que aun cuando el símbolo de la Malinche es parte del discurso de las mujeres migrantes en Estados Unidos: "la india intérprete, no ha sido reapropiada para empoderar las demandas de mujeres indígenas y pobres en el interior de la nación mexicana, salvo en el caso del movimiento zapatista" (210). La observación se aplica a las indígenas, pero puede incluir a las mujeres vulnerables en todo México. En 1995, Elena Poniatowska, interviniendo desde el centro de México, comparaba la escritura de mujeres mexicanas y chicanas, opinando que estas últimas son portadoras de las raíces culturales e históricas mexicanas y se han adelantado para crear un espacio de mexicanidad transgresora. Mientras que en México, afirma:

Nosotras las mujeres nunca hemos dicho, por ejemplo, que somos mujeres de color como lo hacen las chicanas, nunca nos hemos definido como mestizas, blancas o prietas, nunca hemos sabido reclamar nada, y cuando lo hemos hecho ... nos han tildado de locas. ... Las chicanas, en cambio, cruzaron tres mil kilómetros de frontera que nos separan del país más poderoso del mundo con la Virgen de Guadalupe, la Malinche, La Llorona, la Coatlicue a cuestas y allá les dieron un nuevo valor y una identidad que antes no tenían. (47-48)

En su reflexión para comprender las razones que suscitan este fenómeno de distanciamiento entre ambas, Claire Joysmith observa que las propuestas de mestizaje: "are, ironically, often perceived in (central) Mexico as unsettling, even threatening, particulary when they are gender related and when they demand that we seriously and pragmatically rethink mobile cultural identities" ("Anzaldua's Bordercrossing" 49). Considera que las reacciones de resistencia pueden resultar exacerbadas en la academia quizá por un interés de mantener la significación de los iconos míticos femeninos tal como los conocen, o bien, podría deberse a 
prejuicios culturales, religiosos o lingüísticos, incluso a un miedo inconsciente a la transgresión o a una propuesta teórica con la que no se han familiarizado (48-49). Las ideas de López, Belausteguigoitia, Poniatowska y Joysmith exponen una visión general acerca de la literatura de mujeres mexicanas, no específicamente de escritoras de la frontera norte de México o de Ciudad Juárez, quienes definen en su discurso posturas contestatarias contra la violencia. Este trabajo se aleja de teorizar respecto a dicha problematización, pues no establece un desarrollo comparativo o propone perspectivas dominantes entre las dos literaturas revisadas. Sus opiniones, sin embargo, se consideran útiles aquí como antecedente para repensar y considerar diálogos entre ambos lados culturales y literarios.

REESCRITURA DE LA MALINCHE TRAICIONADA EN LOS ESPACIOS DE VIOLENCIA

Hasta ahora, existen numerosas versiones acerca del espíritu errante de una mujer que se aparece llorando y buscando a sus hijos muertos. Una de las leyendas asocia a La Llorona con la Malinche, indígena maya, quien pena por los hijos que tuvo en su relación con el español Hernán Cortés durante la Colonia, a los cuales ella misma asesinó y abandonó. El vínculo con el conquistador pone a la Malinche en la posición de traidora frente a su pueblo de origen, según las primeras interpretaciones históricas de este relato.5 Sin embargo, para las chicanas, quienes lo resignifican, dicho episodio las condenó por ser hijas bastardas, pero incidió finalmente en la conformación de una identidad propia y un "tercer espacio", favoreció la definición de la "cultura mestiza" (Los Movimientos 79). En palabras de Anzaldúa, son ellos, su pueblo, quienes la vendieron, "son las costumbres que traicionan. La india en mí es la sombra: la Chingada, Tlazolteotl, Coatlicue. ${ }^{6}$ Son ellas que oímos lamentando a sus hijas perdidas" (80). Desde entonces, agrega, "es esclava, mano de obra barata, colonizada ... invisible y no ... escuchada" (80). Este planteamiento refuta el juicio y rechaza la culpa que la sentencian como traidora, pues acepta la función de una figura positiva por ser intermediaria entre dos culturas, traductora de sus respectivos códigos y no subordinada a un sujeto de poder, tal como afirman Carlos Monsiváis, Jean Franco y Margo Glantz, en La Malinche, sus padres y sus hijos, editado por esta última autora.

En la poesía y la narrativa de la frontera norte de México, específicamente de Ciudad Juárez, así como en la chicana, hay reiteradas referencias a un escenario fronterizo afectado por los afanes del sistema capitalista y a las secuelas del consumismo, en un espacio de contraste donde conviven el Primer y el Tercer Mundo. Cientos de mujeres en situación económica precaria viajan desde distintos pueblos de México 
para buscar trabajo en las fábricas procedentes del extranjero, o con el propósito de encontrar la oportunidad de cruzar hacia Estados Unidos persiguiendo el difundido "sueño americano". Este deseo, como registra la literatura, se queda atrapado en las orillas de Ciudad Juárez, en sus periferias y perímetros de peligro. En el peor de los casos, la ilusión es irrumpida por el final de sus cuerpos violentados, desmembrados como el de Coyolxauhqui y arrojados en el desierto. 7

Por asociación de las prácticas de la Malinche con el contexto presente, se establece popularmente que el destino funesto de las mujeres en la franja fronteriza es la consecuencia de prestar su cuerpo y su trabajo a la empresa del extranjero económicamente más poderoso, al estadounidense que coloniza el territorio mexicano a partir de la instalación de las fábricas maquiladoras y la contratación de mano de obra latinoamericana a muy bajo costo. El término peyorativo "malinchista" (Octavio Paz, El laberinto de la soledad, 1950) se emplea precisamente para nombrar a quienes repiten este comportamiento de traición a la propia cultura. La complejidad para romper esta dinámica de abusos radica en que las obreras se entregan a dicha labor por necesidad, obligadas por lo que podría considerarse el abandono de un gobierno que no las protege y falla. A este gobierno Cristina Rivera Garza lo ha llamado un "Estado sin entrañas", el cual, por afanes neoliberales, "rescinde su relación con el cuidado del cuerpo de sus constituyentes" (II). ${ }^{8}$ La contestación de las víctimas es otro resultado de la intromisión del capitalismo que Sayak Valencia define como "gore", según el cual los sujetos marginados en territorios fronterizos participan mediante estrategias de violencia en esta urgencia por acceder al modo de vida del Primer Mundo y formar parte de sus prácticas de consumo:

...se crea de esta manera subjetividades capitalistas radicales que hemos denominado sujetos endriagos y nuevas figuras discursivas que conforman una episteme de la violencia y reconfiguran el concepto de trabajo a través de un agenciamiento perverso, que se afianza ahora en la comercialización necropolítica del asesinato ... (19)

Las mujeres se ven involucradas en esta subjetividad cuando también en medio de situaciones de pobreza y frustración ejercen resistencia, participan en alguna actividad destinada a competir, adquirir capital y reafirmar su status social, intervienen en el sistema destructor del cual finalmente terminan siendo víctimas mortales.

Dentro del corpus literario, la mexicana Araceli Bobadilla escribe desde una voz femenina dirigida frontalmente a su dominador: “¿Eres tú 
mi verdugo? / Gusto en conocerte. / Soy Joaquina / tu víctima [...] Planché mi vestido azul / no tiene aberturas / lustré mis zapatos negros / me gusta que reflejen la luz de los semáforos / descolgué mi chamarra gris / es nueva / maquillé mi rostro / aún parece de niña" (I6-I7). Por un lado, se presenta como amante, deliberadamente expuesta para ser seducida y, por otro, expresa una actitud resignada frente a la esclavitud y el asesino. Es el antecedente de una voz que sin llegar a oponerse acepta su vulnerabilidad, podría así estar aceptando o no la condición de víctima. Una clave para entender esta forma de proceder responde, en términos de Jean Franco, a que "la subversión de las mujeres fracasa porque el poder las seduce. Esta es finalmente la lección de la Malinche" (179). Responden a la misma necesidad de entrada a los beneficios del Primer Mundo, según la propuesta plateada por Valencia sobre el "capitalismo gore".

Más explícito es el poemario Querida fábrica (2012), de la escritora juarense Dolores Dorantes, pues se invierte el discurso de las mujeres victimizadas mediante una escritura que erotiza la relación de las obreras de Ciudad Juárez con la maquiladora. La voz femenina enunciadora ama e interpela a su verdugo manifestándole su voluntad de seguirlo: "Fábrica, estoy en el formato / produzco lo que soy / Aunque ese anillo caliente que me rodea las manos me atormente / te sigo / Querida mía / solo tengo tu hambre de cobalto" (54). La fábrica representa al sujeto dominante y se convierte en el verdugo, pero al mismo tiempo seduce porque resguarda las posibilidades de acceso a los deseados privilegios económicos y sociales del Primer Mundo. Así, la obrera descrita en el poema es consciente de su esclavitud y, no obstante, admite su voluntad de entrega, ejerce como amante de este sistema capitalista que se instala por la fuerza en un territorio todavía incapaz de asimilarlo.

Mientras plantea esta convicción, la voz poética en Querida fábrica hace efectiva una estrategia de supervivencia bajo el aplastante engranaje de la máquina; constituye, de acuerdo con la teoría feminista de Donna Haraway, una cyborg, oprimida, pero con una conciencia de diferencia, preparada para resistir las condiciones tecnológicas del Primer Mundo. De Cherríe Moraga, en Loving in the War Years, Haraway toma el ejemplo de reescritura del relato sobre la Malinche para resignificar la construcción chicana de la identidad y expone que "...la raza bastarda enseña el poder de los márgenes y la importancia de una madre como la Malinche. Las mujeres de color la han transformado $\mathrm{y}$, de ser la madre diabólica del miedo masculinista ha pasado a ser la madre letrada original que enseña a sobrevivir" (Haraway 303). La autora se refiere a la capacidad de la mujer para adaptar su cuerpo al organismo industrial y tecnológico de poder. En este caso la mujer sometida al control patriarcal, masculino, y al orden de 
la maquiladora, adquiere habilidades para interactuar fluctuando entre las diversas reglas según le resulte conveniente para salvarse. Además, Haraway propone el rechazo a la victimización mediante una "escritura cyborg" que funciona como descodificadora de los mitos colonizadores occidentales, subvirtiendo el lenguaje dominador (300), como ocurre en los citados textos de Bobadilla y Dorantes.

El llanto que se produce posteriormente sería un efecto del comportamiento de la mujer que en el Tercer Mundo se incorpora a la dinámica del capitalismo agresivo o "biocapitalismo", donde, escribe Morini "es la vida de los individuos la que se vuelve capitalizable" (29). Se actualiza en este discurso de una Malinche que asume las consecuencias violentas de su supuesta traición, pero cuyo lamento no reproduce la culpa, sino, como las chicanas, expresa el abandono o, en el sentido inverso, la traición que ejerce contra ellas el Estado, el sujeto masculino opresor y la indiferencia de otras mujeres hacia la agresión.

ERRANCIA Y VOCES SILENCIADAS SOBRE EL INTERSTICIO

La imagen fantasmal de La Llorona, mujer dolorida que ronda entre las calles o las orillas del río, se reproduce en la literatura sobre Ciudad Juárez bajo la errancia de las madres que buscan a sus hijas perdidas, exigen justicia, o bien, de aquellas mujeres muertas que vuelven en espíritu para encontrar explicación y consuelo. Pero este lamento no se expresa en tono plañidero, sino que es una manifestación armada de sentido reivindicador. A través del llanto, el sujeto femenino impone su presencia sobre el espacio que la relega, se instala como una aparición incisiva y recurrente contra el olvido.

En el relato titulado "Amiga Vera" (20I2), de Elia Hatfield, la desaparecida "Vera deambula por el cerro del Cristo negro" (I9). Hay dos voces enunciadoras: la de la madre que sigue escuchando por las noches a su hija muerta de dieciséis años y la de la joven que observa a esta madre desde su invisibilidad:

Sonrío cuando veo a mamá caminar deambulante por este desierto, sé que su único objetivo es volver a verme. Ahí hay algunas flores que mamá deposita sobre el olor de mi sangre porque mamá intuye que estoy aquí, me habla, me canta canciones de cuna, llora y me repite una y otra vez que vuelva. (22)

La conexión con la imagen simbólica de La Llorona, instalada en el imaginario cultural, es evidente en esta cita en la que aparece la figura de la madre angustiada, revisitando un lugar de vínculos afectivos para llamar a su hija muerta y desaparecida. Al mismo tiempo se describe la presencia 
fantasmal de ésta, la hija, cuyo espíritu se encuentra todavía vagando entre el espacio familiar, en medio de un panorama de confusión. Su andar es, en principio, un acto solitario y pasivo en un espacio indeterminado, donde se ponen en escena el dolor y la memoria.

Primero, el dolor, así como el duelo, se experimentan de manera íntima y privada. El lamento de la víctima, ya sea de la muerta o de la madre, se expresa para comunicar y buscar respuestas; sin embargo, se aísla antes de ser percibido o escuchado por el resto de testigos de la violencia. En los poemas leídos, la mujer asesinada vuelve desde la muerte para materializarse a través de una voz individual que pronuncia un estado de incertidumbre: "Ahí estaba yo / en medio de la más ininteligible de las soledades / circulaba en mi cuerpo un aroma a perro libre / mi propia mortalidad se evaporaba en la indignación / ociosa / ¿qué podía hacer? / ¿Lamentarme? / ¿Horrorizarme? / ¿Consolarme?” (Bobadilla 2I). Esta experiencia se traduce como un intersticio simbólico donde el cuerpo extraviado y la voz se encuentran atrapados, conforma el espacio fronterizo al que Anzaldúa se refiere como un lugar no seguro o de encierro, en el que la mujer (chicana) se halla sometida, "petrificada" entre su propia cultura y la cultura dominante (Los Movimientos 77). Reflectada sobre el terreno literario en torno a las mujeres asesinadas en Ciudad Juárez, dicha frontera se extiende en el plano de abandono y subyugación generado por la violencia patriarcal y machista, junto con la que ejerce el Estado incapaz de evitar o castigar los crímenes.

Micaela Solís señala este escenario de indiferencia social en el que los testigos se hacen sordos a la voz de los lamentos, mermando la posibilidad de trascender la franja en la que las mujeres permanecen errantes:

El espíritu reptil de los testigos que enmudecen,

su sinuoso desliz / por la raíz putrefacta del sistema.

Fronda y semilla el grito afónico en la calle vacía.

Péndulo del miedo sin contrapeso y sin espacio,

destiempo para los últimos compases del peligro,

aterrada la garganta, cerrado el pecho. (43)

El silencio o, más bien, silenciamiento, es una significación de este espacio indeterminado en el que las voces de las víctimas están presentes, pero son ignoradas. El temor que las oprime les impide moverse y arremeter contra el agresor. Antes, durante y después del crimen, las mujeres están impedidas para hacerse escuchar. En palabras de Arminé Arjona: "Nacen desgarradores los gritos del silencio / surgen de la impotencia / crecen indiferentes / agonizan y mueren. / Y no tocan a nadie, su presencia no 
duele / se esconden tras la noche / mas no son percibidos / la gente siempre duerme" (26).

Las autoras coinciden al inscribir dichas voces acalladas que en conjunto forman una atmósfera fantasmal como el que rodea a la imagen mítica de La Llorona, a quien los espectadores miran desde lejos y con espanto. Según los textos, el silencio anida en la ciudad de noche, entre las calles vacías donde huele a muerte, en la apatía de la gente con miedo, la cual enmudece aun siendo testigo de la violencia, y debajo de la tierra donde nadie puede escuchar la súplica de las muertas. En "Poemas por Ciudad Juárez" (2014), la escritora de Tijuana, Rosina Conde, indica la presencia y la voz ignorada de las mujeres que atraviesan el espacio ya como espectros: "Un grito apagado se esparce por el viento de Juárez / desierto plagado de pendones / testigos mudos del silencio / guijarros acompasados por el soplo del rastro que dejaran / las huellas de una ninfa / incapaz de convertirse en laurel" (I9I). Vivas o no, sus pasos y gritos se describen constantemente en los poemas como un murmullo apenas perceptible.

Entonces, los espíritus de las mujeres vagan insistiendo sobre la atrocidad que todos ven, pero de la que casi nadie se ocupa, y, pese a todos los silencios, eso que de ellas resta, de su cuerpo violentado y desaparecido, permea los espacios cotidianos sobre la ciudad y sus alrededores, como apunta Solís: "Estas muchachas de maquila, / tan oscuras en su cabello desgreñado, / tan jóvenes en su muerte, / tan esenciales en el horror. ... / Y estos cerros de Juárez oliendo / a adrenalina y semen. / Y estos baldíos oliendo / a sudor, / adrenalina / y semen" (73). El ambiente, igual que saturado de los olores de la muerte, retiene los pesados lamentos de las mujeres en el aire, de manera que la ciudad misma se personifica en sujeto femenino o madre que llora incapaz de proteger a "sus hijas" del maltrato y del feminicidio.9 Así mismo, la multiplicidad de mujeres asesinadas, las voces que se suman para exclamar dolor, toman de lleno espacios significativos o vitales de este lugar: "En tu desierto, / florece muerte / mujer y llanto / llanto y mujeres ... Llora la tarde / sangre en el cielo / pinta el rojo / atardeceres" (Arjona 27). En contraposición con el silencio y la indiferencia retenida entre los sujetos obnubilados en sus rutinas de la ciudad industrializada, se impone un dolor demasiado real y punzante, que inevitablemente impera sobre el paisaje y obliga, por lo tanto, a mirarlo de frente. El desierto que se traga a los cuerpos y los desintegra va devorando las calles desde la periferia hacia el centro, finalmente "la ciudad está descuartizada" (2I). El sol enrojecido y el atardecer describen un lugar en ruinas habitado por fantasmas, el cual difícilmente puede ignorarse. 
Este paisaje, junto con el dolor invasivo que se instala sobre las dinámicas cotidianas, hace notoria la presencia de las víctimas, aun con sus gritos reducidos a murmullos. Los versos en el poema constituyen una iniciativa solidaria para traer al centro las voces negadas; sus palabras son irrupción de las muertas y desaparecidas, las representan o las encarnan: "Por las quinientas desaparecidas, / -las imaginadas en todas las formas de la muerte-. / Esos fantasmas irredentos / que musitan con las ráfagas del viento: 'Aquí estoy...' / cuando el dolor / cuando el dolor / cuando el dolor" (Solís I9). La escritura de las mujeres en nombre de las víctimas representa así un primer gesto práctico y público, entra con determinación frente al miedo y a la indiferencia, desafía al silencio para hacer explícito el dolor y legitimar el llanto.

Desde esta situación de apertura, en el lugar de voces silenciadas y acumulación de lamentos, madura la reflexión de las mujeres en su búsqueda de contestaciones, no solo respuestas acerca de la desaparición y la muerte, sino con el propósito de encontrar fundamentos para una nueva identidad transgresora, capaz de oponer resistencia contra el dominio masculino, patriarcal y capitalista. En el silencio, durante el primer momento, en el ejercicio privado de la escritura y el reconocimiento del dolor, se preparan para cruzar sus límites y gritar hasta constituirse en sujetos femeninos conscientes de su subjetividad y dispuestas a practicar su empoderamiento. En el intersticio, escribe Anzaldúa, Coatlalopeuh, madre que precede a la figura de La Llorona, "espera con ella: Aquí en la soledad prospera su rebeldía. / En la soledad Ella prospera" (Borderlands/ La Frontera. The New Mestiza 45). ${ }^{\text {.0 }}$ Los elementos de este escenario de errancia y silencio reescriben la figura de La Llorona cuya presencia es el espíritu castigado y sufriente, privado de voz. El segundo rostro de ésta representa al arquetipo de la madre rebelde, la que emite su llanto como grito y convierte su búsqueda en un acto creativo y visible.

BÚSQUEDA Y LLANTO COLECTIVO

El proceso de búsqueda de mujeres desaparecidas en Ciudad Juárez implica movimiento y exige interacción, comunicación con el Otro, exposición y riesgo. En los poemas y relatos de mujeres vulneradas por la violencia son más numerosas las voces que claman por la justicia y la memoria de las muertas o desaparecidas, en comparación con las voces silenciadas mencionadas anteriormente. Primero se enuncia desde una posición individual, cuando el espíritu aparece para reafirmar su presencia, su lugar en el mundo, aun por encima de la muerte: "Soy la que regresa para darles la corrosiva flor de mi memoria, / ... La que pide como única justicia posible: / Ni perdón ni olvido" (Solís 34). Instalada y errante 
en el entorno donde fue violentada, según se abordó antes, el llanto irrumpe con mayor fuerza en el espacio social. Antígona González (2016), poemario de Sara Uribe, es uno de los textos más recientes acerca de la actual violencia que afecta a México. En él apunta a este reconocimiento de las víctimas, señalando su invisibilización y, así mismo, la persistencia de su voz entre los vivos impasibles: "una habitante de la frontera / ese extraño lugar / ella está muerta pero habla / ella no tiene lugar pero reclama uno desde el discurso / ¿quieres decir que va seguir aquí sola, hablando en voz alta, muerta, hablando a viva voz para que todos la oigamos?" (27). Uribe, tras la voz de las "Antígonas" que buscan el cadáver de un hermano o una hija desaparecida, registra numerosas voces errantes en un lugar de ruinas donde los vivos y los muertos son negados y borrados del centro hegemónico político, social y cultural. Insiste en exigir la recuperación del cuerpo para asignarle un lugar fijo, la sepultura en la que pueda configurar la realidad, hacerla inteligible aun con el dolor que conlleva, y sobre la cual pueda mantenerse a salvo el espíritu de los muertos, pero también el espíritu del que tiene la posibilidad de enterrarlo y continuar la vida: "Ellos dicen que sin cuerpo no hay delito. Yo les digo que sin cuerpo no hay remanso, no hay paz posible para este corazón. Para ninguno" (28). El que realiza la búsqueda interpela constantemente al espíritu del desaparecido; sin embargo, el reclamo incesante del cuerpo, las incógnitas sobre su paradero, mantienen esta situación de errancia o aprisionamiento $\mathrm{y}$, por lo tanto, de búsqueda. El llanto requiere la presencia del cuerpo para manifestarse, pues sin esta certeza no hay posibilidad de duelo. Antes, el lamento es una súplica que espera para poder llorar: "Rezo para que tu cuerpo ausente no quede impune. Para que no quede anónimo. Rezo para tener un sitio a donde ir a llorar" (36).

Ahora, el llanto de las mujeres y por las mujeres es un lamento que se vuelve acto consciente, se afirma involucrando a Otros, en este caso a Otras, y se introduce en el espacio político. En ese sentido surge una búsqueda solidaria y colectiva. La testigo es aquí quien retorna para hablar, igual que el espíritu de las desaparecidas. Es, por lo tanto, también una víctima, capaz de empatizar con el dolor, de superar el miedo, dispuesta a sumarse a la búsqueda: "Sublimada en deseo / regreso / por aquellos que recorren las faldas de los cerros ... Los que buscan / al lado de los padres, / la identidad amada de la hija / en la basura / que el viento ensarta en los gatuños" (Solís 24). En este poema se difumina la identidad de la que vuelve, pues el "regreso", que correspondería a la muerta, es la expresión de la viva, quien ocupa el lugar de la Otra imposibilitada para hacerse escuchar. Esta voz que habla en nombre de las desparecidas es recurrente en la escritura de Solís: "Transfigurada en canto / vengo desde 
la zona donde nada significa / emerjo / desde el hálito contrario de la inercia / del poema, / para decirles a todos que morí de una muerte colectiva" (30).

En este sentido, se identifica la declaración abierta del dolor como un elemento significante dentro del ejercicio de transgresión de los sujetos femeninos, cuando el proceso de duelo deviene en una experiencia de reconocimiento y cuestionamiento de sí mismas, pues como señala Judith Butler, el duelo revela al que se queda sufriendo la pérdida aquellos aspectos del Otro que lo constituyen (48). Trasciende la práctica solitaria y "permite elaborar en forma compleja el sentido de una comunidad política ... . Si mi destino no es original ni finalmente separable del tuyo, entonces el 'nosotros' está atravesando una correlatividad a la que no podemos oponernos con facilidad..." (48-49).

Conde, partícipe de esta comunidad, confiesa su llanto en "Poemas por Ciudad Juárez", en el cual reitera la palabra "lloro" para enumerar las razones de su lamento en medio de la violencia sobre el espacio juarense. La autora habla desde un dolor experimentado en forma individual, aunque provenga de un mal que perjudica directamente a Otras: "Tanto coraje reprimido / tanta palabra amordazada / tanto grito en la arena. / No es necesario estar en el ruedo para sentir la estocada / para experimentar el dolor del torturado / para vivir la impotencia por el hijo o por la hermana muerta" (193). La empatía, así como la "vergüenza" del crimen, "de no dar mi vida por ellas," (190), la involucran en un lamento que sumado a otras voces forma parte de un discurso colectivo y político. La voz de Conde llama a la sociabilidad y a la humanización; la violencia hacia las mujeres la incluye, como a todas, porque la sitúa en el mismo escenario de vulnerabilidad, agrediéndola también. En ese sentido, escribe: "Simplemente lloro, / cuando me doy cuenta de mi impotencia; / cuando me dicen que es absurdo llorar por nada; / cuando me preguntan por qué lloro si estoy viva; / cuando la vida pasa y todo es lloro ..." (189).

En un texto solidario que se pronuncia contra la indiferencia y el olvido de la violencia, Rivera Garza escribe sobre la necesidad de reconocer el dolor ajeno implicándose desde los propios sentidos, es decir, resalta la urgencia de "dolerse" y "condolerse". Enfocada particularmente en el contexto de violencia en México, la autora reflexiona junto con otros ensayistas acerca de la experiencia del horror, tan expuesto públicamente que los testigos no pueden quedarse al margen ocupando el lugar de espectadores. "Dolerse", especifica Rivera Garza, proviene de "la necesidad política de decir 'tú me dueles'” (I4). Después del impacto del horror, agrega, ha optado por la palabra para decir: "aquí, tú nosotros, nos dolemos" (I5). Esto es, como sostiene a lo largo de su ensayo, un ejercicio 
político que pretende hacer decible lo indecible, construir un escenario nuevo posible.

La escritura misma es una respuesta al silencio y un discurso que elabora sentidos en medio de un mapa indescifrable por los vacíos que deja la violencia. El llanto se atreve a exponerse abiertamente cuando alguna mujer se responsabiliza del dolor de la Otra: "Déjanos a nosotros continuar el grito no escuchado, / el dolor de esta solidaridad extemporánea, / la violencia de seguir existiendo. / Y, acaso, / danos en una ola, el dulce rumor de tu descanso" (Solís 76). A las que ya no están se les concede el descanso desde la justicia y la memoria, pero éstas que escriben dan continuidad a sus voces y búsquedas mediante llanto, canto $o$ escritura; ejercen el acto político de trasformación para configurar nuevas formas de estar, desde un cuerpo y una subjetividad libre.

En este sentido, la suma de los llantos se abre con la potencia de un grito. Más que un lamento, alberga la intención de intervenir, recuperar los fragmentos de todas las pérdidas que conforman el espacio fronterizo citado por Anzaldúa. En este intersticio, las voces de las mujeres dolidas por la violencia de Ciudad Juárez se preparan para resistir.

EL GRITO Y LA RECUPERACIÓN DEL ESPACIO FEMENINO CREATIVO

En el contexto literario representado sobre Ciudad Juárez, asumir el dolor propio y empatizar con el dolor de los demás es, en cierta manera, ir al encuentro de La Llorona, es decir, aceptar el miedo a la Madre terrible, la que "abandona y castiga", en palabras de Blanca Solares (40). Dicho arquetipo en el imaginario mexicano y chicano está ligado a Coatlicue, diosa mayor en el México antiguo." Significa, en ese caso, reconciliarse con la Madre, enfrentarse y comprender, al mismo tiempo, la orfandad, el trauma del abandono y la culpa por un sometimiento que se repite de diversas maneras durante siglos.

Los nuevos sujetos femeninos recuperan, como las chicanas, los relatos de opresión heredados de la cultura patriarcal y, así pues, la Madre terrible es, en su reinterpretación, "destructora y creativa" (Solares 386), "diosa y monstruo", "benefactora y amenazante", como señala Rebolledo (50). Coatlicue transfiere sus capacidades de supervivencia a sus hijas. Anzaldúa se refiere, entonces, a un "Coatlicue State", es decir, "un preludio para cruzar" (Borderlands/La Frontera. The New Mestiza 70). La conexión de las escrituras de mujeres de la frontera norte de México y chicanas con la imagen de Coatlicue y su reconfiguración en La Llorona, aparece conformando las identidades femeninas transgresoras. En otros textos del corpus chicano se identifican personajes que experimentan la violencia pero la afrontan conscientes de su lugar en el espacio de vulnerabilidad. Se 
presentan relatos que reescriben explícitamente la imagen de La Llorona, en los que el llanto rompe en grito, o bien, donde la actitud de la figura femenina empoderada modifica el comportamiento de los sujetos que la rodean. Sobresalen, por citar ejemplos, el cuento "El arroyo de la Llorona" (1996), de Sandra Cisneros, y el capítulo décimo de la novela Tan lejos de Dios (1996), de Ana Castillo. En el primero, de Cisneros, la personaje llamada Cleófilas, una mujer en Estados Unidos, hija de mexicanos y educada en la tradición patriarcal, habita junto a un arroyo llamado "La Llorona" (Woman Hollering Creek) del cual siempre se pregunta si lleva tal nombre refiriendo dolor o coraje. Oprimida en la casa de los padres y luego maltratada por su esposo, estando embarazada y con un hijo pequeño, recibe ayuda de Felice para escapar. En su huida cruzan el arroyo, momento en el cual Cleófilas imita el grito que Felice, chicana soltera e independiente, acostumbra a dar siempre que atraviesa el puente. El llanto adquiere así la potencia para convertirse en grito y libera a la mujer de su atadura, justo en este espacio simbólico del trayecto posterior al "Coatlicue State", que Anzaldúa explica como el espacio de encierro, de miedo que alberga la resistencia, y que conecta a la mujer con la energía de la Madre, mientras la prepara para cruzar y comenzar la vida (Borderlands/La frontera. The New Mestiza 68-73). El espíritu de La Llorona es recuperado por Cleófilas, transformada en una madre irreverente; con el grito, a diferencia del llanto, impone su voz y se asegura de ser escuchada, pero, sobre todo, se libera de la represión por la que abandona el espacio del matrimonio machista.

Hay una interpretación similar en la novela de Castillo, en la que una familia de mujeres observa a una de las hermanas comportarse de manera extraña. También formadas moral y culturalmente en un espacio de dominio patriarcal, reconocen, igual que el resto del pueblo, las habilidades de esta mujer a la que llaman "Loca", quien, en su rutina de paseo junto al río, puede comunicarse con los animales, manejar situaciones que nadie puede soportar, bordar, cocinar e, incluso, hablar con la mujer que se aparece descalza y vestida de blanco, de quien todos intuyen que es La Llorona, según su conocimiento de la leyenda de origen mexicano. Mientras que las hermanas sufren constantes desilusiones, Loca es la única que tiene la capacidad de mantenerse al margen de los problemas y de ser feliz. Parece que La Llorona le transmite saberes útiles de supervivencia. De tal manera, posee otra de las condiciones que Anzaldúa define como "la facultad", una capacidad para ver fenómenos debajo de la superficie de la realidad, la sensibilidad mediante la cual los oprimidos, los perseguidos, los atrapados entre dos mundos, desarrollan un sexto sentido para detectar el peligro y mantenerse a salvo (Borderlands/La Frontera. La 
Nueva Mestiza 85). Sin dialogar conscientemente con esta propuesta, las escritoras de la frontera norte de México, que representan a las mujeres de Ciudad Juárez, recrean la figura de una mujer que rompe diversas barreras de su cotidianidad, tanto de la frontera física, como de las normas sociales y morales a las que están sometidas. La mexicana Elia Hatfiel, en "La llorona que deambula por el Río Bravo" (2012), relata cómo una mujer se arriesga a cruzar el río desde México hacia Estados Unidos, llevando a su hija en brazos. La madre desafía a los oficiales de la Border Patrol, quienes al ver su cabello largo y despeinado, vestida con falda larga y cargando a una niña que no para de llorar, se retiran creyendo que se les aparece La Llorona. Ella no se propone asustarlos, pero es su actitud, al ocultar su propio miedo, lo que juega en su favor frente a la temida vigilancia. Como en el caso de Felice y de Cléofilas, el coraje revierte al llanto y convierte a las mujeres en dueñas de sí mismas y de su porvenir. Esta manifestación de La Llorona es, finalmente, la versión que enfatiza su fuerza creativa. Según la interpretación de Clarissa Pinkola Estés, La Llorona es un lamento que llama a las mujeres a mantener su propia fortaleza y creatividad. Las acerca a la mujer salvaje (5I2-517).

Conde recupera el imaginario mítico precolombino para describir el escenario de las mujeres asesinadas, fragmentadas corporalmente como Coyolxauhqui. Detrás de éstas renombra a la mujer revestida con los poderes de la diosa, la Madre terrible que espera para levantarse de la muerte, recuperar el cuerpo y la voz, y ayudar a las Otras a recobrarlos:

Hastiada de recoger brazos y piernas;

de amortajar cabezas,

la Supermujer vomitó el polvo que había tragado del desierto, polvo mágico que la mantenía ante la Amenaza Sangrante.

No sabía si los poderes le alcanzarían para unir los

miembros de las Coyolxauhquis norteñas,

ni si llegaría a salvaguardar a las hembras

que florecían en las plantas industriales. (189-190)

La esperanza de las poetas está en el grito solidario de las mujeres. Dos finales optimistas sentencian lo que está por venir: en la última página de Querida Fábrica, Dorantes escribe sobre el margen derecho: "Tu boca antes de reventar:" (63). La frase termina en dos puntos, dejando abierto un presagio, la imagen de una voz que está a punto de hacer erupción. En la última página de Elegía en el desierto, Solís escribe en el centro: “Dichoso será el tiempo de la Mujer de Luz!" (80), cuando aquellos lamentos encuentren lo que buscan. 
La revisión de textos literarios, teóricos y críticos de la literatura de mujeres de la frontera norte de México y chicana revela que, efectivamente, la imagen simbólica de La Llorona está presente como figura de resistencia. Ciertamente, aparece como un referente explícito en la literatura chicana a partir de los textos de Anzaldúa, cuyos planteamientos guiaron la interpretación del discurso de las mujeres de la frontera norte de México. Así, fue posible identificar significaciones de esta figura de origen precolombino asociadas al llanto, a las experiencias de opresión y procesos de transgresión de las víctimas de la violencia en Ciudad Juárez.

Las autoras de la frontera norte de México, quienes escriben sobre Ciudad Juárez, representan, por un lado, al sujeto femenino victimizado en el espacio de la violencia que se produce por el poder patriarcal, la tradición machista y la fractura entre el capitalismo y el Tercer Mundo. Su identidad está emparentada simbólicamente con la doble cualidad de la Malinche, juzgada de traición y a la vez preparada para resistir la opresión. Sobresale el vínculo con la figura de La Llorona al reproducir el estado de errancia, o incertidumbre, el silenciamiento de las mujeres, así como la voz que interpela a Otros quienes, por miedo, ignoran el lamento y la súplica. Por otro lado, en una identidad contestataria, la figura de La Llorona se representa a través de la manifestación abierta del dolor, primero individual y luego colectivo, asumido por las mujeres a través de la escritura, como un acto de empoderamiento y ejercicio político. Y, finalmente, su imagen se recupera mediante la expresión del llanto que deviene en grito, estado en el cual las mujeres reconocen el poder sobre sus voces y sus cuerpos, y deciden transgredir el orden, siguiendo el llamado colectivo de la fuerza creativa que transmite simbólicamente la Madre terrible para su supervivencia.

Si bien el análisis ha posibilitado la puesta en diálogo de textos de escritoras chicanas y de la frontera norte de México, es visible en el corpus mexicano la intención de elaborar un discurso solidario con el dolor, contra el silencio y el olvido, más que el propósito de enfatizar referencias que sustenten la configuración de identidades femeninas empoderadas y subjetivas frente al sistema que las oprime. Sin embargo, aun cuando la representación de La Llorona no es explícita en sus textos, se reescribe en los pasos y la voz de las mujeres que se suman a la resistencia colectiva y, en ese sentido, su escritura encuentra puntos de conexión con las voces de las autoras chicanas. Por último, surge la reflexión dirigida a responder si están presentes y qué significación tienen para dichas escritoras los elementos simbólicos femeninos asociados a la historia nacional y a sus mitos, o bien, cuáles rasgos serían claves para elaborar un sujeto femenino 
que, como a las chicanas, les permita constituir nuevas identidades configuradas desde la conciencia de libertad de sus voces.

Pontificia Universidad Católica de Chile

\section{NOTAS}

* Este artículo se realiza en el marco del proyecto de investigación postdoctoral No. 3150075: "El sujeto femenino en las literaturas de las fronteras MéxicoEstados Unidos. Una perspectiva bifronteriza", financiado por el Fondo Nacional de Desarrollo Científico y Tecnológico de Chile, con patrocinio de la Pontificia Universidad Católica de Chile.

I En "Anzaldúa's Bordercrossing into Mexico", Claire Joysmith expone que el trabajo de Anzaldúa (traducido al español en 2019) se lee rara vez en México dentro de los circuitos académicos u otros (47). Anteriormente, en Las formas de nuestras voces: Chicana y Mexicana Writers in México, citaba "ese infamiliarity with each other's work", del que hablaba Ana Castillo, refiriéndose al desconocimiento o ignorancia de las obras de unas y otras, favorecido por la falta de "traducciones, difusión, conocimiento y disponibilidad" (36).

2 La "crítica biborderlands o bifronteriza" planteada por Antonio Cárdenas en Mar, desierto y ladrillo (2004) y desarrollada, entre otros, por Graciela Silva Rodríguez y Manuel de Jesús Hernández-G, propone incluir los espacios culturales y literarios de ambos lados, en vez de fomentar discursos críticos unilatierales, chicano y mexicano fronterizo: "cada uno estudia y se limita a interpretar la producción cultural y literaria surgida en su respectivo lado fronterizo; raro es el texto crítico que relaciona e interrelaciona de manera extensa y profunda las dos producciones fronterizas" (Silva Rodríguez y Hernández-G 55).

3 La producción literaria y artística (cine, pintura, fotografía, teatro, música, performance) chicana con referencias a La Llorona es muy extensa. Para este estudio se seleccionan textos literarios de algunas autoras. ¿necesario poner esto en nota?

4 Luz Aurora Pimentel analiza la figura de Coatlicue, "símbolo de la alteridad" en México. Compara la visión estética de Justino Fernandez y filosófica de Octavio Paz. Por un lado representa lo que Fernández distiguió en ella: la armoniosidad y racionalidad que le confieren belleza y la convierten en obra de arte. Por otro lado, la inicialmente demonio y luego diosa es, en términos de Paz, un cúmulo de detalles diversos, sincretismo de elementos del cuerpo, aspectos culturales y signos procedentes del universo precolombino, greco- 
romano, moderno, constituyendo en sí misma un conjunto de textos. Coatlicue se convierte así en un poderoso icono de identidad cultural.

$5 \quad$ En su estudio sobre La Llorona en la cultura popular chicana, Domino Renee Perez analiza un extenso corpus literario, obras pictóricas y cinematográficas para identificar formas de su representación, concluyendo que tanto los relatos tradicionales como la mayoría de los contemporáneos, a diferencia de Anzaldúa, la promueven como una figura amenazadora, cuya identidad la reduce a mala mujer y madre (23).

6 Octavio Paz explica que la Chingada es "la Madre violada" y la asocia con la Conquista, "que también fue una violación, no solamente en el sentido histórico, sino en la carne misma de las indias. El símbolo de la entrega es la Malinche, la amante de Cortés. Es verdad que ella se da voluntariamente al conquistador, pero éste apenas deja de serle útil, la olvida. ... Y del mismo modo que el niño no perdona a su madre que lo abandone para ir en busca de su padre, el pueblo mexicano no perdona su traición a la Malinche” (94).

7 Coyolxauhqui, involucrada en la muerte de su madre Coatlicue, fue asesinada en venganza por el dios Huitzilopochtli, su hermano. Para Alicia Gaspar de Alba, es la primera víctima del feminicidio en México; el ritual de su decapitación y desmembramiento recrea la tortura de los cuerpos femeninos de las mujeres en Ciudad Juárez (132).

$8 \quad$ El "Estado sin entrañas" es, según Rivera Garza (2015), "el Estado neoliberal mexicano", que "le ha dado la espalda a sus compromisos y a sus responsabilidades, rindiéndose ante la lógica implacable, la lógica, literalmente letal, de la ganancia" (II).

9 “Las hijas de Juárez" es el título de la exhibición presentada en el Social and Public Art Resource Center (SPARC), realizado en Venecia, California, 2003 (Gaspar de Alba I45). A partir de entonces la frase se ha difundido colectivamente para referirse a las mujeres asesinadas en Ciudad Juárez.

Io Deidad indígena precolombina que Gloria Anzaldúa define como "la que tiene soberanía sobre las serpientes" o "la que se encuentra en armonía con las bestias" (Borderlands/La Frontera. La nueva mestiza 72); según su interpretación, es símbolo "de lo femenino, el movimiento sinuoso de la sexualidad, la creatividad, la base de toda energía y de toda vida” (80). En términos de Irene Ballesteros, para las chicanas es "su guía para descolonizar su cuerpo, su sexualidad y su mente del pasado patriarcal y empoderarse así de la situación para llegar a obtener una existencia anticolonial que le permita reconocerse a sí misma y a los otros" (391-92).

II Coatlicue, "la de falda de serpientes", es la deidad de los aztecas, asesinada por sus hijos ofendidos, quienes la acusaron de deshonra por el nacimiento de Huitzilopchtli, engendrado de una pluma que se introdujo en su vientre mientras barría. 
OBRAS CITADAS

ANZ Al D U A, GL OR I A. Borderlands/La Frontera. The New Mestiza. San Francisco:

Aunt Lute Books, 2007.

—. Borderlands/La Frontera. La nueva mestiza. Trad. Carmen Valle. Madrid: Capitán Swing, 2016.

—. "Los movimientos de rebeldía y las culturas que traicionan". Otras

inapropiables. Feminismos desde las fronteras. Eds. bell hooks, Avtar Brah, Chela Sandoval y Gloria Anzaldúa. Trad. Rocio Macho Ronco et. al. Madrid: Traficantes de sueños, 2004-7I-80.

A RJona, A miné. Juárez, tan lleno de solo y desolado. Chihuahua: Chihuahua Arde editoras, 2004 .

ARRIOLA, ELVIA R. "Accountability for Murder in the Maquiladoras. Linking Corporate Indifference to Gender Violence at the U.S.-Mexico Border". Making a Killing. Femicide, Free Trade and La Frontera. Eds. Alicia Gaspar de Alba y Georgina Guzmán. Austin: U of Texas P, 2010. 25-6I.

B ALLESTER BUIGUES, IRENE. El cuerpo abierto. Representaciones extremas de la mujer en el arte contemporáneo. Gijón: Ediciones Trea, 2012.

B ELA USTEG Uigoitia, MARisa. "Rajadas y alzadas: de Malinches a comandantes. Escenarios de construcción del sujeto femenino en México". Miradas feministas sobre las mexicanas del siglo XX. Coord. Marta Lamas. México, D.F: Consejo Nacional para la Cultura y las Artes/Fondo de Cultura Económica, 2007. I9I236.

в о Ba Dilla, ARACEli. Soy grito. México, D.F.: Cuadernos de Comunicación Sindical, $2 \mathrm{OI} 4$.

B U t L R, JU D ith. Vida precaria. El poder del dueloy la violencia. Trad. Fermín Rodríguez. Buenos Aires: Paidós, 2006.

CAPU TI, JANE. Afterword: Goddess Murder and Gynocide in Ciudad Juárez". Making a Killing. Femicide, Free Trade and La Frontera. Eds. Alicia Gaspar de Alba y Georgina Guzmán. Austin: U of Texas P 2010, pp. 279-294.

CASTAÑEDA SHULAR, ANTONIA, TOMÁS YBARRA-FRAUSTO Y JOSEPH SOMMERS, co m P s. Literatura Chicana. Texto y Contexto. Englewoods Cliffs: Prentice-Hall, Inc., 1972.

castillo, ana. Tan lejos de Dios. Trad. Flavia Company. Barcelona: Muchnik Editores, 1996.

CISNEROS, SA NDRA. El arroyo de la Llorona y otros cuentos. Trad. Liliana

Valenzuela. New York: Vintage Español, 1996.

Conde, Rosina. Poesía reunida. México, D.F.: Desliz ediciones, 2014. Do Rantes, do Lores. Querida Fábrica. México, D.F.: Práctica Mortal, 2012.

F RANCO, JEAN. Las conspiradoras: la representación de la mujer en México. México,

D.F.: El Colegio de México, 2003. 
gaspar de alba, Alicia. "Poor Brown Female: The Miller's Compensation for 'Free' Trade". Making a Killing. Femicide, Free Trade and La Frontera. Eds. Alicia Gaspar de Alba y Georgina Guzmán. Austin: U of Texas P, 2010. 63-93.

-. [Un]Framing. The "Bad Woman". Sor Juana, Malinche, Coyolxauhqui and Other Rebels with a Cause. Austin: U of Texas P, 2014.

G LANTZ, MARGo, coord. La Malinche, sus padres y sus hijos. México, D.F.: Taurus, 2013 .

HARAWAY, D ON NA J. Ciencia, cyborgs y mujeres. La reinvención de la naturaleza. Trad. Manuel Talens. Madrid: Ediciones Cátedra, I99I.

H A TF I L D, E l i A. Por los caminos del norte: relatos de mujeres de la frontera. México, D.F.: Ediciones Eón, 2012.

Joysmith , CLAiRE. "Anzaldua's Bordercrossing into Mexico". Signs: Journal of Women in Culture y Society 37.I (201I): 46-52.

—, E D. Las formas de nuestras voces. Chicana and Mexicana Writers in Mexico. México, D.F.: Universidad Autónoma de México, 1995.

LÓ PE Z, ARA LiA. “Consideraciones para pensar las diferencias entre las escritoras mexicanas y chicanas contemporáneas”. Las formas de nuestras voces. Chicana and Mexicana Writers in Mexico. Ed. Claire Joysmith. México, D.F.: Universidad Autónoma de México, 1995.5I-64.

M E L Ro, PI Lar. Mythological Constructs of Mexican Femininity. New York: Palgrave Macmillan, 2015.

morini, Cristina. Por amor o a la fuerza. Feminización del trabajo y biopolítica del cuerpo. Madrid: Traficantes de sueños, 20I4.

PAZ, о с т A vi o. El laberinto de la soledad. Postdata. Vuelta al laberinto de la soledad. México, D.F.: Fondo de Cultura Económica, 1994.

PEREZ, D O MIN O RENEE. There Was a Woman. La Llorona from Folklore to Popular Culture. Austin: U of Texas P, 2008.

Pimentel, LuZ A UR ORA. "Ekphrasis and Cultural Discourse. Coatlicue in Descriptive and Analytic Texts". Neohelicon 30 (2003): 6I-75.

PINKOLA ESTÉs, CLAR ISSA. Mujeres que corren con los lobos. Trad. María Antonia Menini. Madrid: Punto de Lectura, 2003.

P ONIAT OWSKA, ELENA. "Mujeres Chicanas y Mexicanas". Las formas de nuestras voces. Chicana and Mexicana Writers in Mexico. Ed. Claire Joysmith. México, D.F.: Universidad Autónoma de México, 1995. 45-50.

R E B O L L D o, TE y da NA. Women Singing in the Snow. A Cultural Analysis of Chicana Literature. Tucson: $\mathrm{U}$ of Arizona $\mathrm{P}, \mathrm{1} 995$.

rivera garza, cristina. Dolerse. Textos desde un país herido. México, D.F.: Ediciones, 2015 .

SAntos, CRISTIna. Unbecoming Female Monsters. Witches, Vampires, and Virgins.

Lancham: Lexington Books, 2017. 
Silva rodríguez, graciela y manuel de Jesús hernández G, eds. Chican@sy Mexican@s Norteñ@s: Biborderlands Dialogues on Literary and Cultural Production. México, D.F.: Ediciones Eón, 2012.

So La Re S, B L A N CA. Madre terrible. La Diosa en la religión del México Antiguo. Barcelona: Anthropos, 2007.

So Lís, M IC A L L A. Elegía en el desierto. In memoriam. Ciudad Juárez: Universidad Autónoma de Ciudad Juárez, 2004.

TAB UEnCA CóRdoba, MARía So corro. "Ghost Dance in Ciudad Juárez: at the End/Beginning of the Millennium”. Making a Killing. Femicide, Free Trade and La Frontera. Eds. Alicia Gaspar de Alba y Georgina Guzmán. Austin: U of Texas P, 20IO. 95-II9.

valencia triana, SAyak. Capitalismo gore. Santa Cruz de Tenerife: Editorial Melusina, 20IO.

U I BE, SARA. Antígona González. Los Ángeles: Les Figues Press, 2016. 